\title{
Synthetic enhancer design by in silico compensatory evolution reveals flexibility and constraint in cis-regulation
}

\author{
Kenneth A. Barr ${ }^{1,2^{*}}$ (D), Carlos Martinez ${ }^{3}$, Jennifer R. Moran ${ }^{4,5}$, Ah-Ram Kim6 ${ }^{6}$ Alexandre F. Ramos 7,8 \\ and John Reinitz $1,2,5,9$
}

\begin{abstract}
Background: Models that incorporate specific chemical mechanisms have been successful in describing the activity of Drosophila developmental enhancers as a function of underlying transcription factor binding motifs. Despite this, the minimum set of mechanisms required to reconstruct an enhancer from its constituent parts is not known. Synthetic biology offers the potential to test the sufficiency of known mechanisms to describe the activity of enhancers, as well as to uncover constraints on the number, order, and spacing of motifs.

Results: Using a functional model and in silico compensatory evolution, we generated putative synthetic even-skipped stripe 2 enhancers with varying degrees of similarity to the natural enhancer. These elements represent the evolutionary trajectories of the natural stripe 2 enhancer towards two synthetic enhancers designed ab initio. In the first trajectory, spatially regulated expression was maintained, even after more than a third of binding sites were lost. In the second, sequences with high similarity to the natural element did not drive expression, but a highly diverged sequence about half the length of the minimal stripe 2 enhancer drove ten times greater expression. Additionally, homotypic clusters of Zelda or Stat92E motifs, but not Bicoid, drove expression in developing embryos.

Conclusions: Here, we present a functional model of gene regulation to test the degree to which the known transcription factors and their interactions explain the activity of the Drosophila even-skipped stripe 2 enhancer. Initial success in the first trajectory showed that the gene regulation model explains much of the function of the stripe 2 enhancer. Cases where expression deviated from prediction indicates that undescribed factors likely act to modulate expression. We also showed that activation driven Bicoid and Hunchback is highly sensitive to spatial organization of binding motifs. In contrast, Zelda and Stat92E drive expression from simple homotypic clusters, suggesting that activation driven by these factors is less constrained. Collectively, the 40 sequences generated in this work provides a powerful training set for building future models of gene regulation.
\end{abstract}

Keywords: Gene regulatory models, Even-skipped regulation, Cis-regulatory logic, Transriptional control, Synthetic enhancers, Bicoid, Hunchback, Zelda, Stat92E, Dicheate

\footnotetext{
*Correspondence: kenneth.a.barr@gmail.com

${ }^{1}$ Committee on Genetics, Genomics, and Systems Biology, University of

Chicago, Zoology 111, 1101 E 57th St, 60637 Chicago, Illinois, USA

${ }^{2}$ Department of Ecology and Evolution, The University of Chicago, 60637

Chicago, Illinois, USA

Full list of author information is available at the end of the article
} 


\section{Background}

Enhancers, also known as cis-regulatory modules (CRMs), are DNA segments that recruit sets of sequence-specific transcription factors (TFs) in order to control the spatiotemporal expression of genes. These elements are critical in controlling cell fate in development [1] and are under selection [2-4]. More recently, genetic variation within enhancers has been widely implicated in common human disease $[5,6]$. Predicting the effects of this cis-regulatory variation on local gene expression remains a challenging task. Even in the best studied enhancers, there is evidence of unknown function $[7,8]$, and it is not yet possible to reconstruct these elements from their constituent parts $[9,10]$.

The enhancer which drives the second of seven transverse stripes of even-skipped (eve) in the developing Drosophila blastoderm is among the most studied enhancers in all of biology. A deletion of a $480 \mathrm{bp}$ fragment located $1.1 \mathrm{~kb}$ upstream of the transcription start site leads to loss of this stripe [11], and it is the smallest known fragment sufficient to drive reporter expression in a stripe 2 pattern [12]. Footprinting, TF knockouts [13] and site-directed mutagenisis [14] of this minimal stripe 2 element (MSE2) have identified 4 TFs that act through 12 sites in order to direct the stripe 2 pattern. MSE2 is broadly activated in the blastoderm through the activators Bicoid (Bcd) and Hunchback ( $\mathrm{Hb})$ and forms a stripe through repression by the factors Giant (Gt) on the anterior and Kruppel (Kr) on the posterior [12-15]. Despite being subject to such detailed molecular dissection, there are unexplained features of this enhancer. For instance, deletions of sequences outside the 12 footprinted sites all led to changes in function and additional TFs are required to prevent aberrant expression driven by this enhancer [8].

Enhancers integrate the simultaneous, opposing effects of both activators and repressors in order to determine specific expression levels. Thus, predicting the output of enhancers given any level of input requires quantitative methods. To address this, confocal microscopy has been used to generate spatial and temporal atlases of protein $[16,17]$ and mRNA $[18,19]$ levels at single nucleus resolution during the first $4 \mathrm{~h}$ of Drosophila development. Using transgenesis of enhancers driving reporter expression, the precise input-output function of enhancers can be measured. Sequence-level models (SLMs) of gene regulation have been used to describe this function as an emergent property of underlying TF binding sites [20-27]. Such models predict binding using thermodynamics and incorporate known, context-dependent rules of TF function, such as repression through short-range quenching [28-30]. SLMs have identified additional binding sites, regulators and interactions that are important in the control of MSE2 [20, 25].
Experiments with enhancers across Drosophila species suggest that there is considerable flexibility in the architecture of stripe 2 enhancers. Sequences that have diverged over tens of millions of years still drive stripe 2, despite a lack of sequence conservation [26, 31-34]. This functional conservation in the absence of sequence conservation indicates that there are many ways to construct a stripe 2 enhancer. SLMs trained on enhancer-reporter data from $D$. melanogaster have successfully predicted the activity of stripe 2 enhancers (S2Es) from distant Drosophilids [25] and identified accessible evolutionary paths that conserve expression through compensatory evolution [26]. This suggests that the context-dependent rules incorporated into SLMs are sufficient to describe the flexibility of MSE2 cis-regulatory logic.

While SLMs have been able to successfully describe the activity, evolution and flexible architecture of evolved enhancers, such enhancers represent only a small proportion of the sequences that are predicted to drive stripe 2 [35]. Instead, selection may obfuscate many constraints on the order and arrangement of TF motifs that give rise to functional S2Es by removing nonfunctional motif configurations from natural populations. This is suggested by the fact that sequences that lie outside of known binding motifs are necessary for expression $[7,8]$, as well as by past failures to generate synthetic Drosophila enhancers using reconstituted binding sites $[9,10]$.

Synthetic enhancers offer a potential means to address the extent to which known regulatory mechanisms represent a complete description of the cis-regulatory function, and to uncover hidden constraints on cis-regulatory architecture. While SLMs can be used to generate thousands of sequences that are predicted to drive virtually any pattern along the Drosophila anterior-posterior (AP) axis [35], the apparent success or failure of such sequences to drive the expected expression pattern is uninterpretable in the presence of a large number of changes from naturally selected sequence. Because the large number of sequence changes will tend to conflate those changes that are neutral with those that are critical, what is needed is a method in which functional changes can be attributed to a single or small number of sequence changes. Furthermore, because the minimum requirement for constructing a functional regulatory element is unknown, such changes must be made from the starting point of functional naturally selected sequence.

In this work we introduce a novel approach to the design of synthetic enhancers. Using synthetic compensatory evolution, we generated two series of S2Es with decreasing similarity to MSE2. These series address the extent to which SLMs describe the eve stripe 2 regulatory function and provides informative data when results differ from predictions. In total, we tested the activity of 40 synthetic putative regulatory sequences using site-specific 
integration [36] in developing Drosophila embryos. We collected quantitative expression data from 8 synthetic enhancers. We found that an SLM was able to successfully balance the effects of activators and repressors in order to maintain a stripe even after over a third of binding motifs were lost. We showed that a synthetic sequence half the size of the previously minimal stripe 2 element is able to drive stripe 2 at more than 10 times the levels driven by MSE2. We showed that homotypic arrays of the activators Zelda (Zld) and Stat92E (Dst) are able to drive expression, but activation driven by Bcd and $\mathrm{Hb}$ is sensitive to the spacing, affinity, and orientation of sites. Additionally, we found that motif content not only controls mean expression levels, but also variability in expression within single embryos.

\section{Results}

\section{Design of synthetic enhancers with decreasing similarity to MSE2}

The rational design of synthetic enhancers requires a quantitative model that is able to balance the action of numerous activators and repressors acting on the same DNA sequence. The developing Drosophila embryo permits the input-output function of regulatory DNA to be assayed with single nucleus precision. In this work we placed test sequences upstream of a lacZ reporter using site specific integration in Drosophila embryos so that integration site effects were fixed and output could be quantitatively compared across lines (Fig. 1a). The embryos were subsequently imaged in nuclear cycle 14 (C14), timeclass 6 (T6) $[16,17]$ for nuclei, lacZ and Eve protein (Fig. 1b). At this point in development seven stripes of eve expression are clearly defined, but crossregulation from other pair-rule genes is absent [11,37]. Next, nuclei were segmented (Fig. 1c) and data from multiple embryos were averaged to yield an expression profile for each enhancer in a $10 \%$ wide stripe along the AP axis (Fig. 1d) [38]. We considered nuclei from 35.5 to $92.5 \%$ embryo length, where there is a clean functional distinction between the AP and dorsal-ventral axis. This profile was then registered to an atlas of protein levels [39]. The resulting dataset is a cell by cell assay of transcription under the control of quantified TFs, which can be used to obtain the input-output function of any DNA sequence (Fig. 1e).

In previous work, we generated quantitative models that explain the cis-regulatory function of eve stripe 2 from multiple species. In these works the kinetic parameters of an SLM were trained to the input output function of multiple enhancers (Fig. 2a-b). In one instance, fusions of the Drosophila even-skipped stripe 2 and stripe 3 enhancers gave rise to novel expression patterns [40] that proved a rigorous training set for SLMs. An SLM trained on this data was able to predict expression pattern driven

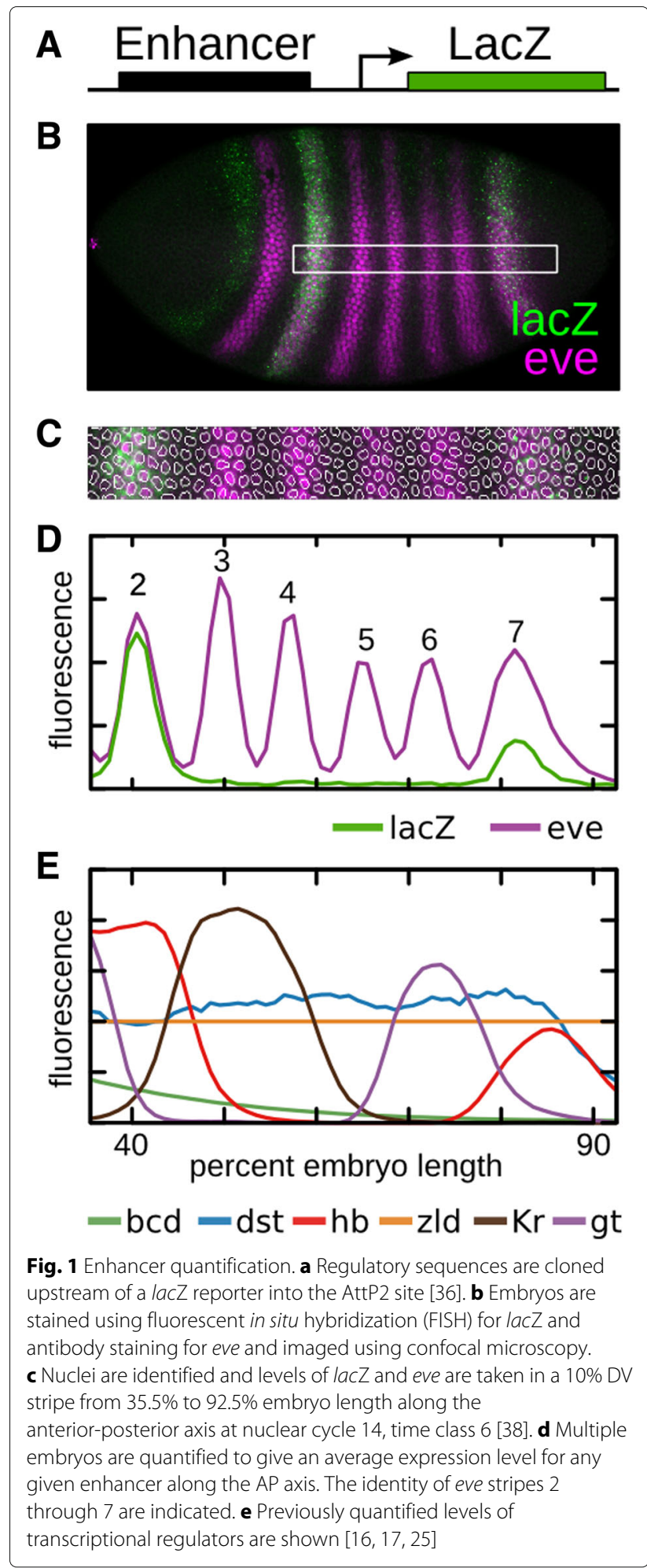

by S2Es from distant Drosphilid and Sepsid flies [25]. In another example, a model trained on S2Es from multiple Drosophilids identified putative ancestral S2Es and accessible evolutionary paths between them [26]. 


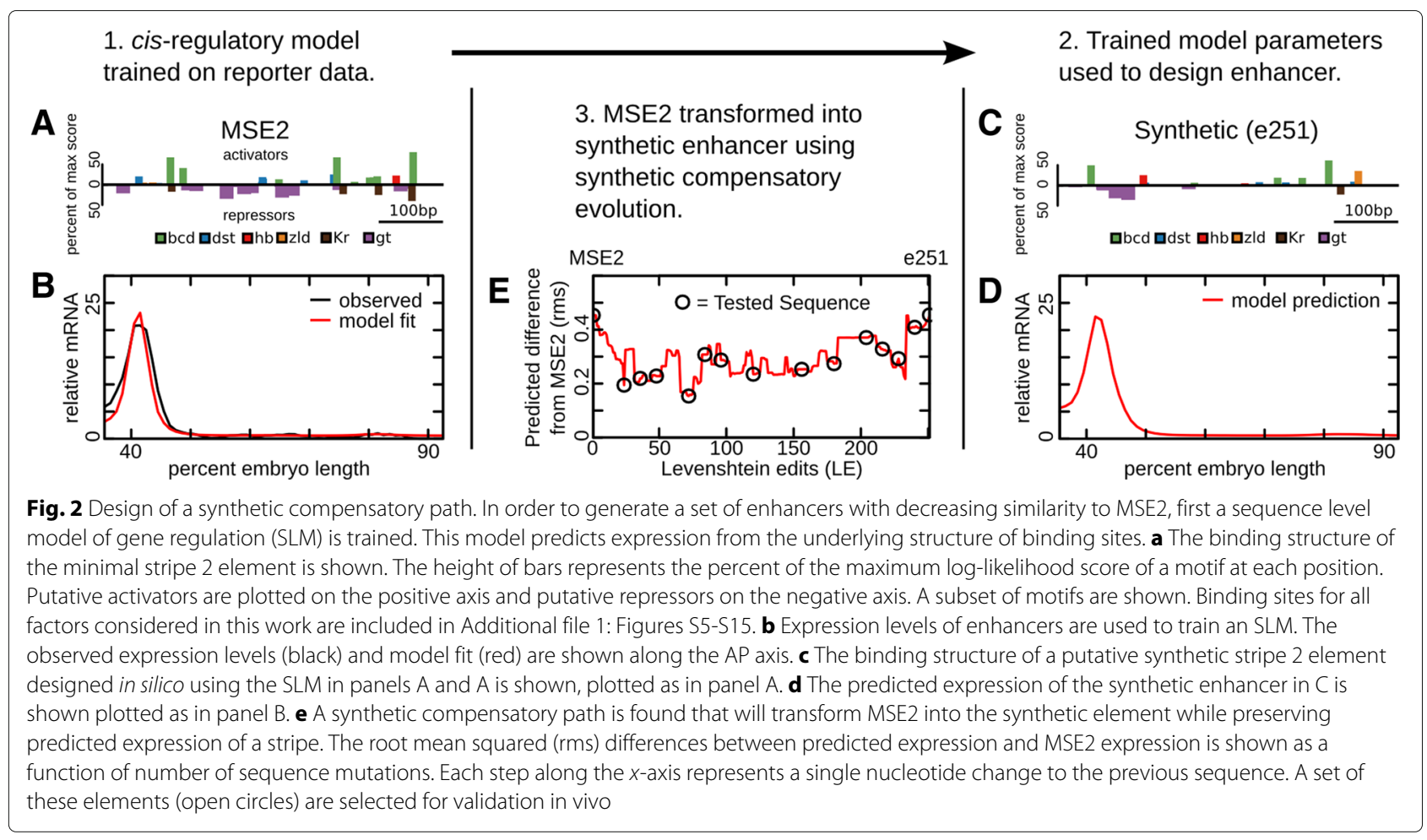

The parameters of SLMs generated in these studies provided a starting point for the design of synthetic regulatory sequences. Keeping the kinetic parameters of the SLMs fixed, we optimized DNA sequence using simulated annealing. We selected sequences that minimized the sum of squared differences between the expression of MSE2 and the predicted expression from one to seven models, each with its own set of kinetic parameters (see Fig. 2c-d and "Methods" section). This process yielded sequences that were designed $a b$ initio to drive expression in the pattern of eve stripe 2 . Two such $a b$ initio enhancers were generated and tested in vivo in the present study.

In order to generate a set of sequences with decreasing similarity to MSE2, we also generated sets of compensatory mutations that mutate MSE2 into each of the $a b$ initio synthetic enhancers while maintaining stripe 2 expression. This was done by finding the single nucleotide changes required to mutate one sequence into the other, known as the Levenshtein edits (LE), and permuting their order such that predicted stripe 2 expression was conserved as much as possible at each edit [26, 35]. We call this set of edits a "neutral path." Each neutral path used the same set of kinetic parameters as were used to design the $a b$ initio synthetic construct at the end of the path. We selected paths of 15 and 11 putative enhancers respectively to test in vivo (Fig. $2 \mathrm{e}$ ).

The two ab initio synthetic enhancers generated in this work are separated from MSE2 by 251 and 272 LE respectively, and we call them e251 and s272. Sequence e251 was designed to drive expression from well separated binding sites using the in silico strategy described above. In contrast, sequence s272 was designed to be a "sub minimal" stripe 2 element that was as short as possible subject to the constraint that all TFs known to regulate stripe 2 could bind and exert their regulatory effects by mechanisms known to operate in S2E. This was done by arranging consensus bindings motifs for known regulators of stripe 2 by hand and then adjusting their affinity such that differences between stripe 2 expression and the model output of this enhancer were minimized. We discuss each of these sequences and the neutral paths in turn.

\section{Expression along the e251 synthetic compensatory path}

The first four sequences tested along the neutral path to e251-at 24 LE (e24), $36 \mathrm{LE} \mathrm{(e36),} 48 \mathrm{LE}$ (e48), and 60 LE (e60) from MSE2-all successfully balanced activation and repression in order to maintain expression of a stripe at $40 \%$ embryo length (Fig. 3). As predicted, each of these four sequences expressed at levels greater than MSE2 (Fig. 3a-e). The remaining 11 sequences at greater than 72 LE did not drive expression in the modeled region. In addition to this region, many embroys also drove expression within the anterior portion of the embryo (Additional file 1: Figure S1A-E). The vector used in this work has previously been reported to drive an ectopic stripe anterior to eve stripe 1 [40]. To confirm that expression is driven by the vector, we generated a control construct that contained no enhancer. Embryos 


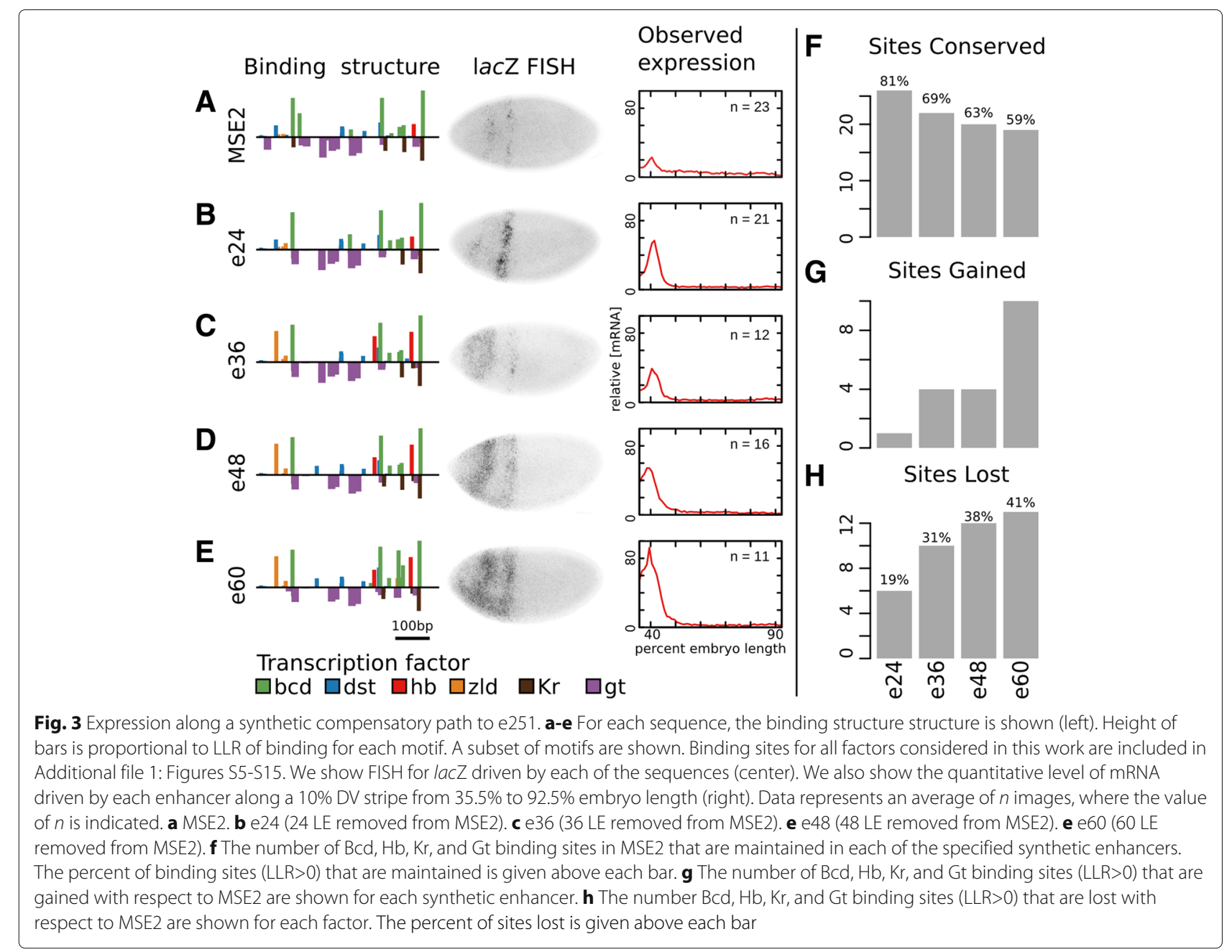

with this construct drove expression of an ectopic stripe (Additional file 1: Figure S2), albeit at levels considerably lower levels than in some tested synthetic enhancers.

To confirm that the sequence changes resulted in binding site turnover, we examined the gain and loss of binding motifs for the key regulators $\mathrm{Bcd}, \mathrm{Hb}, \mathrm{Kr}$, and Gt. We identified a total of 32 binding motifs for these regulators at a log-likelihood ratio (LLR) [41] greater than zero. By 60 LE, 19 (59\%) sites were maintained (Fig. 3f), 10 sites were gained (Fig. 3g), and 13 (41\%) were lost (Fig. 3g). This level of binding site turnover is comparable to that seen between D. mel and D. erecta (Additional file 1: Figure S3), which are several million years diverged [42] and show quantitative differences in expression driven by their respective S2Es [26].

\section{Known motifs cannot explain loss of expression after $60 \mathrm{LE}$} While sequences at $60 \mathrm{LE}$ or less drove stripe 2 expression, sequences at $72 \mathrm{LE}$ or more failed to drive expression. We sought to identify which of the 12 sequence mutations were responsible for this change. Most model parameters predicted a reduction in expression at $72 \mathrm{LE}$ (Additional file 1: Figure S1) as a result of the loss of a $\mathrm{Hb}$ motif and reduced affinity for Bcd. Recent work has highlighted the importance of the lost $\mathrm{Hb}$ site [25], making it a prime candidate for the change which caused loss of expression. The loss of the $\mathrm{Hb}$ motif was a result of a single nucleotide A $>$ T change (ATAAAAA to ATATAAA). We reversed this change in e72 to restore the $\mathrm{Hb}$ motif. This did not rescue expression in e72 (Fig. 4c).

A single nucleotide $T>G$ change from e60 to e72 (GGATTA to GGATGA) disrupted a consensus Bcd motif. $\mathrm{Hb}$, which is typically a repressor, is able to activate when bound near Bcd [25, 40,43]. To test whether the loss of $\mathrm{Bcd}$ and $\mathrm{Hb}$ was responsible for loss of expression at e72, we restored both the Bcd and $\mathrm{Hb}$ sites in $\mathrm{e} 72$. The resulting sequence did not rescue expression driven by e72 (Fig. 4 d).

The remaining 10 nucleotide differences between e60 and e72 do not lead to appreciable differences in the predicted affinity for modeled TFs. We checked for predicted changes in binding preferences for factors within the Fly 


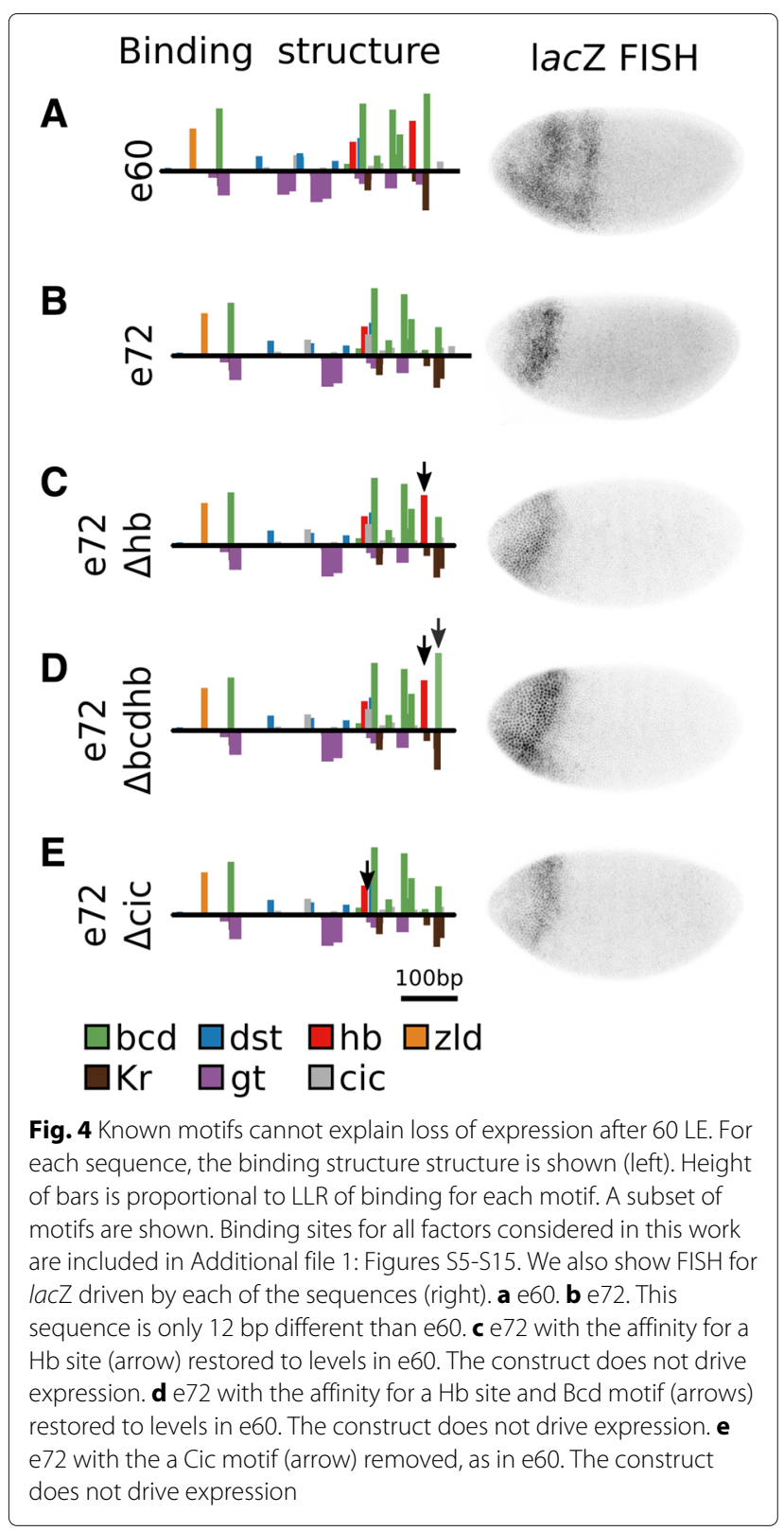

Factor Survey [44] that are maternally or ubiquitously expressed in the Berkeley Drosophila Genome Project [45]. A single candidate emerged. A single nucleotide $T>A$ change (TGATTG to TGAATG) led to the creation of a site for the repressor Capicua (Cic) that is expressed throughout the entire modeled region. This repressor is reported to set borders of Bcd target genes [46], and its binding motif is present in all tested sequences at 72 or greater LE from MSE2. Removal of this site did not restore expression in e72 (Fig. 4e).

\section{Expression along the $\mathbf{2} 272$ synthetic compensatory path}

The sequence $\mathrm{s} 272$ was designed to contain the motifs and interactions currently known to be essential for stripe 2 expression (Fig. 5a). The sequence contains a single motif for Dst, which is known to be essential for expression of eve stripe 3 [47] and is a major activator of zygotic expression [48]. It contains two adjacent motifs for the activator Bcd, which binds to DNA cooperatively [49]. It contains a single $\mathrm{Hb}$ site, which activates expression of eve stripe 2 when bound near Bcd $[20,25]$. These four activator motifs are flanked by two Zld motifs, which has been reported to open chromatin $[50,51]$. Finally, the sequence contains motifs for the repressors Gt and $\mathrm{Kr}$, which set the boundaries of stripe 2 expression [12-15, 20]. In addition to this sequence, we designed and tested the activity of ten sequences in a set of $272 \mathrm{LE}$ that mutate MSE2 into this synthetic enhancer while conserving stripe expression (Fig. 5b, Additional file 1: Figure S4 i).

While the designed enhancer did not drive reporter expression (Additional file 1: Figure S4), two tested sequences drove expression of a stripe at $40 \%$ embryo length. A sequence at $100 \mathrm{LE}$ (s100) drove weak expression of stripe 2, despite having lost 22 of 32 (69\%) of binding motifs for the factors Bcd, Hb, Gt, and $\mathrm{Kr}$ (Fig. 5d). Another sequence, 250 LE from MSE2 (s250), drove very strong expression of a stripe at $40 \%$ embryo length despite having only $2(6 \%)$ motifs conserved with respect to MSE2. At $319 \mathrm{bp}$ in length, with the majority of motifs falling in a less than $200 \mathrm{bp}$ cluster, this sequence is significantly smaller than the 480 bp minimal stripe 2 element, yet drove expression at levels more than 10 times greater than MSE2 (Fig. 5e).

\section{Homotypic clusters of Zelda and Stat92E drive embryonic expression}

The sequence s272 did not drive expression despite being separated by only 22 LE from a strong enhancer. We hypothesized that loss of expression could be due to an imbalance between activation and repression. To test this hypothesis, we generated a variant of the designed enhancer that eliminated motifs for $\mathrm{Kr}$ and Gt (s272 $\Delta t \Delta \mathrm{Kr}$ ). The resulting sequence failed to drive expression (Fig. 6a).

In order to determine which TFs are capable of driving expression alone, we generated sequences with homotypic clusters of 6 motifs for Zld, Bcd, and Dst. Starting from the previous construct, we replaced each of the 6 motifs with a motif for the specified factor, keeping any intermotif sequences constant. Some small differences from consensus motifs were necessary to prevent the creation of repressor motifs. Homotypic clusters of Zld drove moderate expression (Fig. 6b) and homotypic clusters of Dst drove strong levels of expression (Fig. 6d).

\section{Bcd binding orientation is important for MSE2 function} While homotypic clusters Zld and Dst drove reporter expression in developing embryos, $6 \mathrm{Bcd}$ motifs failed to 

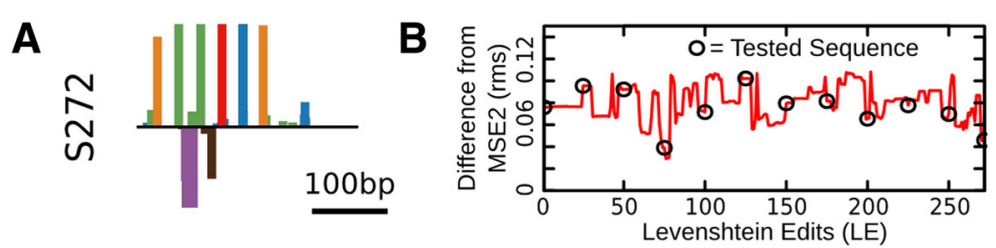

Observed

Binding structure lacZ FISH expression

C


D
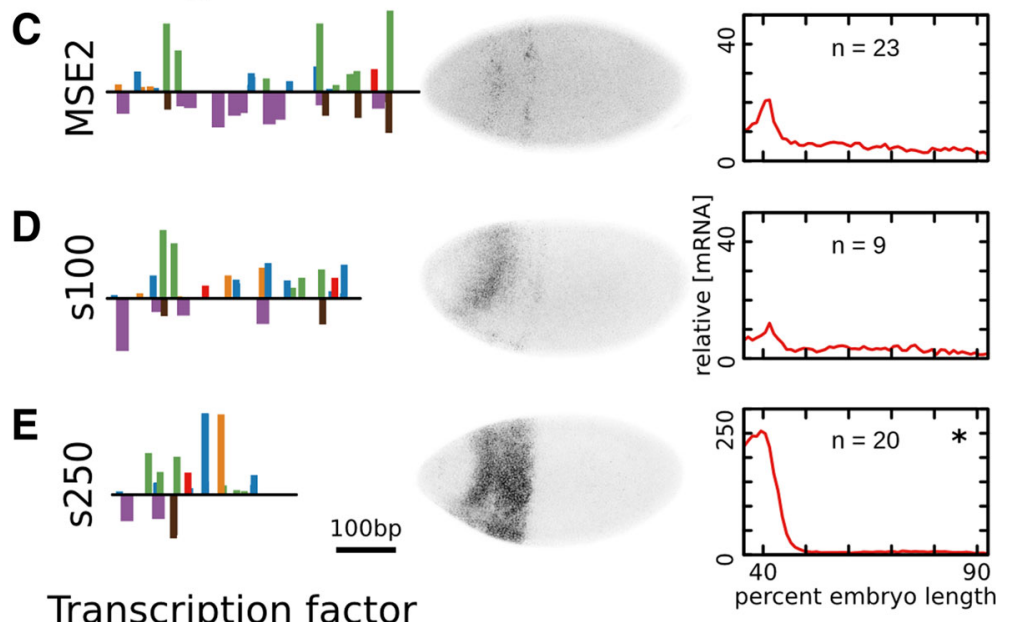

\section{Transcription factor} ubcd $\square$ dst $\square \mathrm{hb} \square \mathrm{zld} \quad \square \mathrm{Kr} \quad \square \mathrm{gt} * \mathrm{y}$-axis rescaled

Fig. 5 A 319bp synthetic enhancer drives stripe 2 at levels more than 10 times greater than MSE2. a The binding structure of a sequence designed, with model feedback, to drive expression of a stripe 2 pattern is shown. Height of bars is proportional to the LLR. Most sites represent consensus motifs for each factor. Binding sites for all factors considered in this work are included in Additional file 1: Figures S5-S15. b The predicted root mean squared differences from MSE2 expression along a series of 272 edits that would transform MSE2 into the sequence in panel A. 10 sequences (open circles) that were tested in vivo are shown. c The binding structure of MSE2 (left). FISH for lacZ driven by MSE2 (center). Quantitative levels driven by MSE2 (right). The number of averaged images, $n$, is indicated. d The binding structure of s100 (left). FISH for lacZ driven by s100 (center). Quantitative levels (right) driven s100, a sequence 100 edits from MSE2. This sequence drives expression of levels slightly less than MSE2. The number of averaged images, $n$, is indicated. e The binding structure of s250 (left). FISH for lacZ driven by s 250 (center). Quantitative levels (right) driven by a s250, a 319 bp sequence 250 edits from MSE2. This sequence drives expression at levels more than 10 times greater than MSE2. The number of averaged images, $n$, is indicated

drive expression (Fig. 6c). The fact that Bcd immunoprecipitation preferentially pulls down sequences with Bcd in the head to head orientation [52] suggests that Bcd orientation may be an important factor. This point is strengthened by the fact that sequences with different $\mathrm{Bcd}$ orientation and spacing drove different levels of expression in fly embryos $[49,53]$. In order to test the role of Bcd orientation in MSE2, we reversed the orientation of two Bcd sites, keeping the affinities of all sites constant (Fig. 6e). The resulting sequence drove significantly lower levels of expression than MSE2 (Fig. 6f).

In order to test the role of $\mathrm{Bcd}$ orientation in s272 $\Delta g t \Delta K r$, we generated a sequence that reversed the orientation of a Bcd motif (Fig. 7a). This sequence did not restore expression. In order to test whether helical orientation on DNA prevented these sites from binding cooperatively, we removed 5bp of inter-motif DNA. This sequence did not restore expression (Fig. 7b). Finally, to test whether $\mathrm{Hb}$ was not being coactivated, and thus preventing expression through quenching, we tested a sequence that reversed a Bcd motif orientation and removed the $\mathrm{Hb}$ motif. This sequence did not restore expression (Fig. 7c).

\section{Bicoid, Huncback, and Dicheate are essential for expression driven by $\mathrm{s} 250$}

While various orientations of $\mathrm{Bcd}$ and $\mathrm{Hb}$ did not restore expression in s272, the sequence s250 drove strong expression despite being only $22 \mathrm{LE}$ different in sequence. The fact that Bcd binding orientation is important in MSE2 suggested that the specific orientation of Bcd and $\mathrm{Hb}$ motifs in s250 is essential. Additionally, a strong binding motif for the TF Dicheate (Dic) was lost between s250 and $\mathrm{s} 272$.

We tested whether each of these changes was responsible for loss of expression individually. Editing a single 




Fig. 6 Homotypic clusters of Zld and Dst, but not Bcd drive embryonic expression. a-d For each sequence, the binding structure structure is shown (left). Height of bars is proportional to LLR of binding for each motif. A subset of motifs are shown. Binding sites for all factors considered in this work are included in Additional file 1: Figures S5-S15. We show FISH for lacZ driven by each of the sequences (center). We also show the quantitative level of mRNA driven by each enhancer along a 10\% DV stripe from 35.5\% to $92.5 \%$ embryo length (right). Data represents an average of $n$ images, where the value of $n$ is indicated. a 2272 with sites for repressors Gt and Kr removed. $\mathbf{b}$ Each motif in s272 $\Delta \mathrm{gtKr}$ was replaced with a motif for Zld, preserving inter-motif sequences. The resulting enhancer drives expression across the entire length of the embryo. c. Each motif in $s 272 \Delta \mathrm{gtKr}$ was replaced with a motif for Bcd, preserving inter-motif sequences. Arrow represent the orientation of binding motifs. The resulting sequence did not drive expression. d Each motif in s272 $\Delta \mathrm{gtKr}$ was replaced with a motif for Dst, preserving inter-motif sequences. The resulting enhancer drives expression across the middle of the embryo. The number of averaged images, $n$, is indicated. $\mathbf{e}$ The binding structure of MSE2 is shown with arrows indicating the orientation of Bcd motifs in the sequence. $\mathbf{f}$ The quantitative expression driven by MSE2 and MSE2 with the motif orientations indicated in panel $\mathrm{E}$ reversed. The resulting sequence has the same predicted affinity for Bcd, but drives less expression than MSE2

$\mathrm{Bcd}$ and single $\mathrm{Hb}$ site and their inter-motif sequence led to a complete loss of expression driven by the sequence (Fig. 8b). Surprisingly, a change of only 3 bp that removes a single motif for Dicheate also led to complete loss of expression (Fig. 8c).

\section{Motif content controls variability in expression within embryos}

We noted a difference in the visual appearance of mRNA fluorescence in situ hybridization (FISH) for expression driven by homotypic clusters of Dst compared to Zld (Fig. 6b, d), which seemed to indicate a high level of variation in expression between adjacent nuclei in the construct driven by Dst. In order to investigate within embryo variation we first adjusted for differences in mean expression between embryos ("Methods" section), and then considered the expression in individual nuclei across the AP axis when driven by homotypic clusters of Dst (Fig. 9a) and Zld (Fig. 9b). The levels of expression driven by Dst appeared to have both a higher mean and greater variability than expression driven by Zld. To test whether the higher mean levels could explain variability, we tested the relationship between the mean and standard deviation in each line. We found that there was a linear relationship between the mean expression and standard deviation of expression in $1 \%$ bins along the AP axis, but the slope 




of this line for the Dst driven enhancer was nearly double that of the Zld driven enhancer (0.28 to 0.54) (Fig. 9c), indicating that greater expression variability cannot be explained by difference in the mean.

In order to observe the shape of the distribution independent of the mean, we divided the fluorescence values by the mean levels at each AP $1 \%$ bin from 60 to $80 \%$ embryo length. The resulting distribution in fluorescence about the mean is wider when driven by Dst than when driven by Zld (Fig. 9e-f).

\section{Discussion}

Synthetic biology affords the opportunity to rigorously test constraints on the number, order, and types of TF binding sites required to drive specific spatial and temporal expression of genes. In this work we generated 40 synthetic sequences using a model of gene regulation that captures known chemical mechanisms and rules that govern the architecture of enhancers. These enhancers, which had varying degrees of similarity to MSE2, were constructed in order to address the degree to which these mechanisms and rules are sufficient to describe the activity of enhancers. We found that while the model successfully predicted the activity of several enhancers,

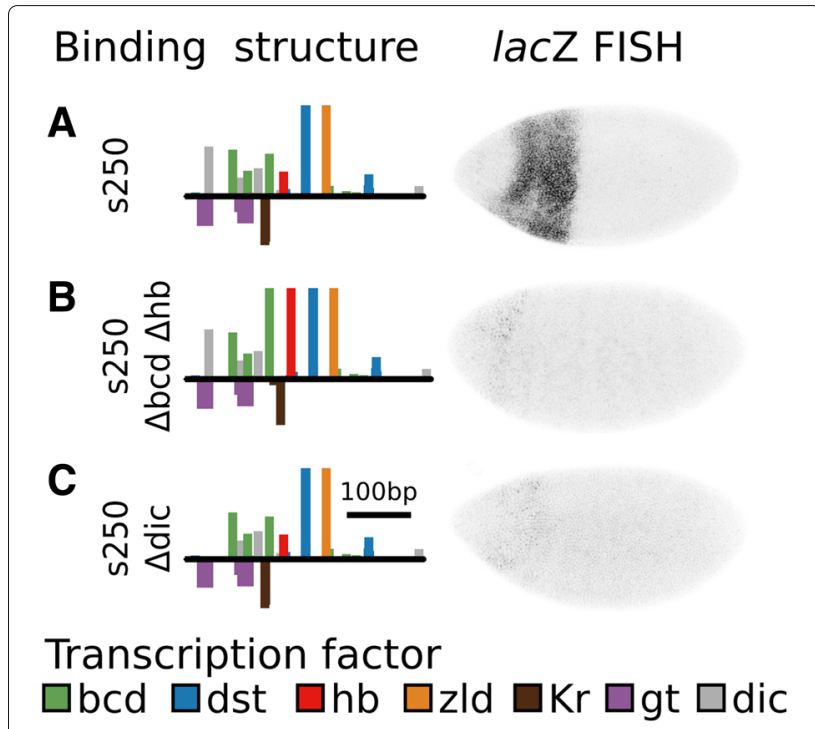

Fig. 8 Bicoid, Huncback, and Dicheate are essential for expression driven by $\mathbf{2 5 0}$. For each sequence, the binding structure structure is shown (left). Height of bars is proportional to LLR of binding for each motif. A subset of motifs are shown. Binding sites for all factors considered in this work are included in Additional file 1: Figures S5-S15. We also show FISH for lacZ driven by each of the sequences (right). a s250. The the factor Dicheate has been included in the binding structure in grey. The enhancer drives strong expression. $\mathbf{b}$ s250 with $\mathrm{Bcd}$ and $\mathrm{Hb}$ orientation reverted to the orientation present in s272. The resulting enhancer does not drive expression. c s250 with the a single Dicheate site removed. The resulting enhancer does not drive expression incongruities point to new molecular players and mechanisms that are required to predict regulatory function.

The mechanisms included in our model are DNA binding, steric competition for DNA binding, cooperative binding, short-range repression, direct repression, and coactivation of $\mathrm{Hb}$ by Bcd or Caudal. This set has been sufficient to explain the flexibility evident in enhancer sequence divergence over the course of evolution $[25,26]$. The fact that S2E expression was maintained in the first tested synthetic compensatory path, even after $41 \%$ of binding sites for key regulators were lost, suggests that these mechanisms explain much, but not all, of the function of S2E.

\section{Additional factors}

Despite this initial success, only the first 4 of 15 tested synthetic sequences in the path to e251 successfully drove expression in nuclear cycle 14. e60 drove expression and e72 failed to drive expression. We analyzed the 12 nucleotide changes that led to loss of expression in e72. Of these 12 changes, 3 were in known binding motifs. Restoring these three changes did not restore expression. This result indicated that there are either unknown motifs which have been gained or lost, or that the edits resulted 

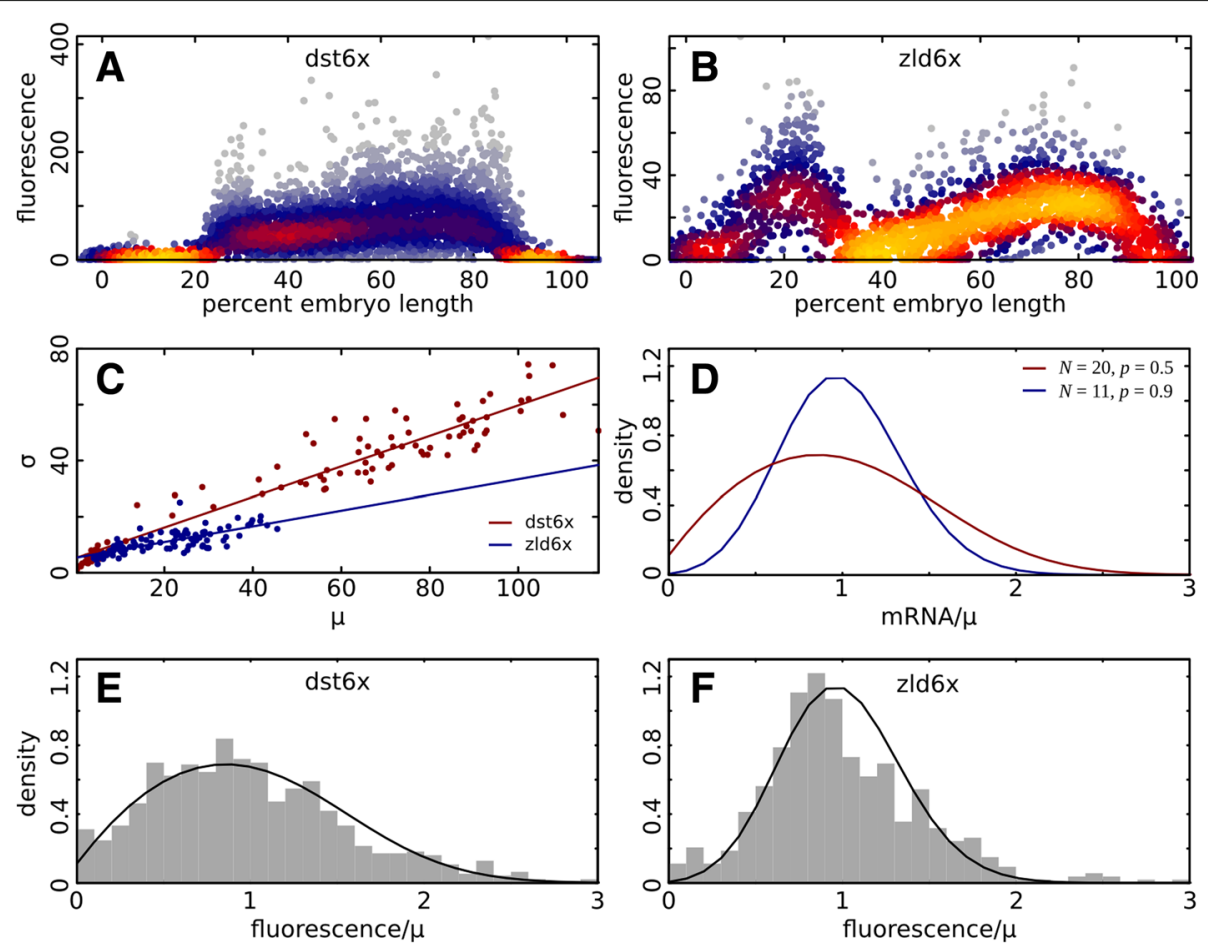

Fig. 9 Motif structure controls variability in expression a Individual nucleus fluorescence levels driven by the construct dst6x. Expression data on each embryo was scaled to minimize the the sum of squared pairwise differences in fluorescence intensity between embryos in $1 \%$ bins along the AP axis. Points are colored according to their local density. $\mathbf{b}$ Expression levels driven by the construct zld6x, plotted as in panel A. c To test whether differences in standard deviation can be explained by different mean expression levels, the standard deviation as a function of the mean expression is plotted for each $1 \%$ bin along the AP axis for both dst6x (red) and zld6x (blue). The relationship between standard deviation and mean is linear with different slopes for each construct. d Two distributions of mRNA count, divided by the mean, in a stochastic transcription model in which the number of transcripts and the ON-OFF state of the promoter are coupled random variables (see Methods). The parameter $N$ represents the strength of transcription when the gene is in the ON state, and $b$ is the probability of finding the gene in the ON state. e Fluorescence values of nuclei were divided by the mean levels in each $1 \%$ bin from 60 to $80 \%$ embryo length. The resulting distribution in fluorescence about the mean for dst6x is shown. The distribution with $N=20$ and $p=0.5$ from panel $\mathrm{D}$ is also shown (black line). e The distribution of fluorescence about the mean for zld $6 x$ is shown. There is a significant difference in the variance of the zld $6 x$ and dst6x distributions (Fligner-Killeen test, $p=1.7 \times 10^{-8}$ ). Additionally, the distribution with $N=11$ and $p=0.9$ from $\mathrm{D}$ is shown (black line)

in other structural changes to DNA that disrupted function.

The result that additional factors regulate MSE2 has been suggested by other work. Andrioli et al. [8] showed that five deletions outside of 12 footprinted sites for $\mathrm{Bcd}, \mathrm{Gt}, \mathrm{Kr}$ and $\mathrm{Hb}$ all disrupted the function of MSE2. Similarly, Vincent et al. [9] showed that an enhancer reconstituted with all 12 footprinted sites failed to drive expression. In addition to these 12 footprinted motifs, this work also modeled non-footprinted sites as well as sites the factors Zld, Dic, Dst, Knirps, and Tailless. Despite considering considerably more putative regulators of MSE2 than these previous works, this list of regulators is likely still incomplete. This is also the case for other other Drosophila enhancers, where function was found to reside within most inter-motif sequences [7, 10]. Alternatively, DNA features such as GC content and dinucleotide content may affect reporter activity through structural effects or by modulating affinity for nucleosomes [54].

\section{Constraints on enhancer architecture}

Every enhancer in the neutral paths considered in this work was constructed to contain a similar balance of bound activators and repressors. Despite this fact, these sequences drove vastly different levels of expression in developing embryos. This suggests that there are additional interactions between bound transcription factors that modulates their activity. We characterized one particular interaction in this work, Bcd cooperativity, that has a constraint not considered in the model. We found that changing the orientation of two Bcd sites within MSE2 disrupted activity of the enhancer, leading us to conclude that pairwise cooperative binding of Bcd requires a pair of sites with opposite orientation on the same strand.

We also discovered a molecularly uncharacterized component of interactions between $\mathrm{Bcd}$ and $\mathrm{Hb}$. In sequences containing only six binding sites for known activators, we were unable to find combinations and orientations of $\mathrm{Bcd}$ and $\mathrm{Hb}$ that drove expression in developing embryos, 
even in the absence of known repressors (Fig. 7), and despite the sequence being compatible with expression when the six motifs were substituted with either Zld or Dst. Despite this, changing 15 nucleotides between s250 and $\mathrm{s} 250 \Delta b c d \Delta \mathrm{hb}$ in such a way as to increase the affinity and spacing of a Bcd and $\mathrm{Hb}$ site was able to render the strongest enhancer assayed in this work non-functional (Fig. 8b). Collectively, this suggests that spacing and orientation of $\mathrm{Bcd}$ and $\mathrm{Hb}$ sites is critical in controlling levels of expression. While there are many permissible configurations, as evidenced by binding turnover in evolution, there may be many more configurations that are not permissible. Further work with synthetic sequences that exhaustively tests regulatory output as a function of distance and orientation will be required to precisely define this interaction.

This conclusion that enhancers are highly sensitive to the arrangement of binding sites is supported by other work with synthetic enhancers. In Drosophila embryos, synthetic sequences containing homotypic and heterotypic clusters of binding sites were sensitive to small changes in intermotif distances and motif orientations [55]. Furthermore, these constraints were tissue dependent, suggesting that changing concentrations of cofactors may affect constraints on cis-regulatory architecture. Similarly, synthetic sequences designed to probe the distance dependency of quenching found that this function is not monotonic [30] and depended on orientation [56]. In mouse, analysis of synthetic sequences containing various complexities of motif structure identified numerous pairwise synergistic interactions [57]. Moreover, synthetic sequences containing eight motifs drove highly variable expression depending on the arrangement [57].

\section{Expression variability}

We found that synthetic enhancers drove different levels of within-embryo expression variability that is independent of the mean (Fig. 9c, e-f). These distributions are reminiscent of distributions seen in a stochastic transcription model in which the number of transcripts and the $\mathrm{ON}-\mathrm{OFF}$ state of the promoter are coupled random variables $[58,59]$. We found that the width of distributions of mRNA levels, scaled to the mean, is altered by varying the probability that a gene is actively transcribing, $p$ (Fig. 9d). While the mean expression level driven by six Dst motifs is far higher than that driven by six Zld motifs, the distribution driven by Zld is less variable than that driven by Dst. This indicates that the the probability that the promoter is in the ON state is lower when driven by Dst than by Zld. This difference could be explained by the role of Zld as a chromatin remodeler. While Dst can drive high levels of transcription, it must compete with nucleosomes for binding. Where Dst has displaced nucleosomes high levels of transcription are achieved, while adjacent nuclei are inactive. In contrast, if Zld can more easily displace nucleosomes, all nuclei will have consistent levels of Zld binding. This leads to a high probability of the gene being in the ON state, even though Zld activates transcription more weakly than Dst.

\section{Conclusions}

Interpreting and predicting the function of regulatory DNA, directly from sequence, remains a fundamental challenges in molecular genetics. It will require understanding the ways in which bound transcription factors interact in order to modulate gene expression. Functional models of gene regulation incorporate known transcription factors and their interactions in order describe and predict the function of regulatory elements.

In this work we tested the ability of a functional model of gene regulation to predict the expression driven by putative synthetic enhancers that have varrying degrees of similarity to MSE2. Initial success indicated that these factor mechanisms explain much of the function of MSE2, however we found evidence for both new factors and interactions that have not been incorporated into previous models. Specifically, we show that orientation of Bcd sites is critical in MSE2 (Fig. 6f), and that the interaction between $\mathrm{Bcd}$ and $\mathrm{Hb}$ is highly sensitive to spacing or affinity (Fig. 8b). In contrast, simple homotypic clusters of Zld and Dst drove expression, indicating that these factors may be less constrained with respect to the spacing and orientation of motifs.

Typically, models of Drosophila gene regulation have been trained on functional enhancers, but it is important to consider both positive and negative data in training sets. The sequences generated in this work contain many instances in which active elements are separated from inactive elements by only a few nucleotides. This property will rigorously constrain future models of gene regulation.

\section{Methods \\ Design of synthetic enhancer sequences}

The method of optimizing sequence given a set of kinetic parameters is discussed in depth in Martinez et al. [35]. In brief, seven parameters sets, characterized in three previous works $[25,26,35]$, were used to generate the sequences in this work. The parameters for these models are given in Additional file 1: Table S1. Parameter sets 1, 2, and 3 are described Kim et al. [25], where they are called model 01,06 , and 07 respectively. Parameter set 4 was trained using all the data from Kim et al. as well as the expanded model in Martinez et al. [26]. This fit used the PWMs for $\mathrm{Hb}$ and Bcd, that are reported in Martinez et al. Parameter sets 5 and 6 were trained to the same data used by Kim et al. but used a different PWM for Bcd [60]. Parameter set 7 was obtained by training with the PWMs and data used in Kim et al., with the addition of Zld as a 
uniformly expressed activator at a constant level of 100 . The Zld and Cic PWMs are from the Fly Factor Survey [44]. All PWMs used in this study are given in Additional file 1.

The synthetic sequence e251 was designed using all seven parameter sets. In order to generate a sequence with well separated sites, we used the cost function

$$
E=\left(\sum_{i}\left(x_{i}-y_{i}\right)^{2}\right)+\beta o,
$$

where $x_{i}$ and $y_{i}$ are the model output and data respectively, $\beta$ is a configurable parameter, $o$ is the number of overlapping motifs, and collectively $\beta o$ is a penalty for overlapping binding motifs. We defined two motifs as overlapping if the end to end distance between their footprints was within 5 nucleotides, and we set $\beta$ to be $1 \%$ of the maximum possible score, corresponding to $x_{i}=255$ for all $x$. To generate e251, we minimized the mean cost function

$$
E_{\text {consensus }}=\frac{1}{7} \sum_{i=1}^{7} E_{i}
$$

where $i$ denotes a parameter set from the set of seven described above. The initial sequence used was random.

The synthetic sequence s 272 was designed by starting with a 258 bp DNA having the structure shown in Fig. 5a, but with each binding site having a maximum affinity consensus sequence. This sequence drove a predicted stripe 2 pattern a few nuclei anterior of the observed pattern when assessed using parameter set 2 . The affinities for repressors Gt and $\mathrm{Kr}$ were then reduced such that the pattern was predicted to drive expression of a stripe at the position of stripe 2 . This was accomplished with a single nucleotide change to the Gt consensus motif (TTACGCAAT to TTACGCAAA) and three changes to the $\mathrm{Kr}$ consensus (TAACCTTTC to AAACCCATTT).

\section{Design of enhancers by synthetic compensatory evolution}

The method of generating synthetic compensatory paths is discussed in depth in two previous works $[26,35]$. In brief, we select the synthetic sequence (e251 or s272), identify the number of single nucleotide edits required to mutate MSE2 into this sequence, then permute the order of edits such that at each step we minimize a cost function. We define the function

$$
F=\sum_{i}\left(\frac{x_{i}}{\max _{j} x_{j}}-\frac{y_{i}}{\max _{j} y_{j}}\right)^{2} \times \text { Penalty, }
$$

where $x_{i}$ is the predicted model output and $y_{i}$ is the data. This function standardizes both data and output on a
0 to 1 scale. We penalize model predicted expression less than data with the multiplicative penalty,

$$
\text { Penalty }=\left\{\begin{array}{ll}
\frac{\max _{i} y_{i}}{\max _{i} x_{i}} & \max _{i} x_{i}<\max _{i} y_{i} \\
1 & \max _{i} x_{i} \geq \max _{i} y_{i}
\end{array} .\right.
$$

For the path to s272, we minimize the the function

$$
F_{\mathrm{s} 272}=\sum_{i=1}^{272} F_{i}
$$

where $i$ denotes the sequence after $i$ LE given the permutation being scored. Only model 2 was used in scoring.

For the path to e251, which uses consensus design, we minimized the function

$$
F_{\text {consensuse251 }}=\sum_{i=1}^{251} \sum_{j=1}^{7} F_{i j},
$$

where $i$ denotes the sequence after $i \mathrm{LE}$ and $j$ is a parameter set from the set of seven previously described.

\section{Generation of reporter constructs}

Reporter constructs where generated using a $\mathrm{pCaSpeR}$ backbone (GeneBank X81644.1) containing the promoter and first 22 amino acids of eve fused to lac Z, generated by Small et al. [40]. An AttB sequence was inserted into the multiple cloning site using the restriction enzyme $X b a 1$ for insertion in the AttP2 landing site on chromosome 3 [36]. The enhancer sequence was extended by PCR primers containing overlap with this vector (Additional file 1). The vector was then digested by enzymes EcoR1 and Xho1 and the enhancer was inserted using Gibson assembly [61]. The resulting vector was injected into flies of the genotype $\mathrm{P}\{$ nos-phiC31 $\backslash$ int.NLS $\} \mathrm{X}, \mathrm{P}\{$ CaryP $\}$ attP2 by Rainbow Transgenics. Quantitative data was collected from these lines as previously described [38].

\section{Sequences used in this work}

The sequences of all 40 enhancers generated in this work are included in Additional file 1. Additionally, expression data are provided in Additional file 2.

\section{Analysis of binding site conservation}

In order to determine the number of binding sites gained, lost, or conserved between two sequences we first performed a pairwise alignment between two sequences using the $\mathrm{R}$ package Biostrings. The log-likelihood ratio (LLR) of binding was calculated at every position in each aligned sequence. Sequences were called binding sites if the LLR was greater than zero. In order to accommodate gaps in sequence alignments, sites were considered conserved if they aligned within $3 \mathrm{bp}$. Sites were considered lost if there was no site with LLR greater than 0 within $3 \mathrm{bp}$ on the corresponding aligned sequence. For the background distribution we use the frequencies of nucleotides 
in the Drosophila genome $\left(P_{\mathrm{bg}}(A)=P_{\mathrm{bg}}(T)=0.297\right.$, $\left.P_{\mathrm{bg}}(C)=P_{\mathrm{bg}}(G)=0.203\right)$.

\section{Scaling of data for variation analysis}

Variation in expression can be due to effects both within and between embryos. In order to remove the between embryo effects, we introduced a scaling factor for each embryo which multiplies the fluorescence measurements across the entire AP axis. We then optimized the scaling factors for each embryo in order to minimize the sum of squared differences in fluorescence measurements between embryos of the same genotype. This was subject to the constraint that the sum of scaling factors equals the number of embryos of that genotype. The scaled data from multiple embryos was then pooled for subsequent analysis.

\section{Theoretical distribution of mRNA}

The steady-state distribution of mRNA counts has been previously derived for a stochastic transcription model in which the number of transcripts and the ON-OFF state of the promoter are coupled random variables ([62], Eq. 29). This distribution is defined by three variables: $p$ gives the probability of the promoter being in the ON state, $N$ gives the transcription rate when the promoter is in the $\mathrm{ON}$ state, and $b$ gives the rate of switching between $\mathrm{ON}$ and OFF states. Ramos et al. ([62], Eq. 29) gives the distribution of mRNA when the promoter is in the OFF state, $\alpha_{n}$, or ON state $\beta_{n}$. Here we report the total distribution $\phi_{n}=\alpha_{n}+\beta_{n}$, keeping the parameter $b$ fixed at $b=4$. The mean number of mRNA is given by $\mu=N p$.

\section{Additional files}

Additional file 1: Supplementary Materials. A PDF containing supplementary figures, tables, position weight matrices used, and sequences generated in this work. (PDF $4800 \mathrm{~kb}$ )

Additional file 2: Quantified expression patterns. An xls file containing averaged fluorescence measurements of the expression pattern driven by the enhancer sequences used in this work. (XLS 664 kb)

\section{Abbreviations}

Bcd: Bicoid; C14: Nuclear cycle 14; Cic: Capicua; CRM: cis-regulatory module; Dic: Dicheate; Dst: Drosophila Stat92E; eve: even-skipped; FISH: Florescence in situ hybridization; Gt: Giant; Hb: Hunchback; Kr: Kruppel; Kni: Knirps; LE: Levenshtein edits; LLR: Log-likelihood ratio; MSE2: The even-skipped minimal stripe element 2; rms: root mean squared; S2E: Stripe 2 element. Any natural or synthetic sequence that drives the even-skipped stripe 2 pattern; T6: Timeclass 6 ; TF: Transcription factor; Zld: Zelda

\section{Acknowledgements}

We would like to thank Pengyao Jiang, Misha Ludwig, Jackie Gavin-Smyth, Manu, and all other members of the John Reinitz and Martin Kreitman labs for helpful discussion. We thank Unjin Lee and the students of BIOS 21410 for their help in enhancer synthesis.

\section{Funding}

This work was supported by the National Institute of Health R01 OD010936 and the University of Chicago. The funders had no role in the study design, data collection and analysis, decision to publish, or preparation of the manuscript.
Availability of data and materials

All data generated or analyzed during this study are included in this published article and its supplementary information files.

\begin{abstract}
Authors' contributions
KB designed all synthetic enhancer sequences, collected all the reporter data, and wrote the manuscript. CM and ARK developed the model of gene regulation and trained it to data. JRM designed the reporter vector assisted in the generation and cloning of synthetic enhancers. AFR generated the two state transcription model. JR conceived of the study design, supervised the work, and edited and revised the manuscript. All authors read and approved the final manuscript.
\end{abstract}

Ethics approval and consent to participate

Not applicable

Consent for publication

Not applicable

\section{Competing interests}

The authors declare that they have no competing interests.

\section{Publisher's Note}

Springer Nature remains neutral with regard to jurisdictional claims in published maps and institutional affiliations.

\section{Author details}

${ }^{1}$ Committee on Genetics, Genomics, and Systems Biology, University of Chicago, Zoology 111, 1101 E 57th St, 60637 Chicago, Illinois, USA. ${ }^{2}$ Department of Ecology and Evolution, The University of Chicago, 60637 Chicago, Illinois, USA. ${ }^{3}$ Department Biochemistry and Molecular Genetics, Northwestern University, 60611 Chicago, Illinois, USA. ${ }^{4}$ Department Human Genetics, The University of Chicago, 60637 Chicago, Illinois, USA. ${ }^{5}$ Institute for Genomics \& Systems Biology, The University of Chicago, 60637 Chicago, Illinois, USA. ${ }^{6}$ School of Life Science, Handong Global University, 37554 Pohang, Gyeongbuk, South Korea. ${ }^{7}$ Departamento de Radiologia - Faculdade de Medicina, Universidade de São Paulo \& Instituto do Câncer do Estado de São Paulo, São Paulo, 05403-911 SP CEP, Brazil. ${ }^{8}$ Escola de Artes, Ciências e Humanidades \& Núcleo de Estudos Interdisciplinares em Sistemas Complexos, Universidade de São Paulo, Av. Arlindo Béttio, 1000 CEP 03828-000, São Paulo, SP, Brazil. ${ }^{9}$ Department Statistics, The University of Chicago, 5747 S. Ellis Avenue Jones 312, 60637 Chicago, IL, USA.

Received: 5 July 2017 Accepted: 9 November 2017

Published online: 29 November 2017

\section{References}

1. Levine M. Transcriptional enhancers in animal development. Curr Biol. 2010;20:R754-63.

2. Ludwig MZ, Bergman CM, Patel NH, Kreitman M. Evidence for stabilizing selection in a eukaryotic enhancer element. Nature. 2000;403:564-7.

3. Andolfatto P. Adaptive evolution of non-coding DNA in Drosophila. Nature. 2005;437:1149-53.

4. Ward LD, Kellis M. Evidence of abundant purifying selection in humans for recently acquired regulatory functions. Science. 2012;337:1675-8.

5. Hindorff LA, Sethupathy P, Junkins HA, Ramos EM, Mehta JP, Collins FS, et al. Potential etiologic and functional implications of genome-wide association loci for human diseases and traits. Proc Natl Acad Sci U S A. 2009;106:9362-7.

6. Maurano MT, Humbert R, Rynes E, Thurman RE, Haugen E, Wang H, et al. Systematic localization of common disease associated variation in regulatory DNA. Science. 2012;337:1190-5.

7. Swanson CL, Evans NC, Barolo S. Structural rules and complex regulatory circuitry constrain expression of a Notch- and EGFR-regulated eye enhancer. Dev Cell. 2010;18:359-70.

8. Andrioli LPM, Vasisht V, Theodosopoulou E, Oberstein A, Small S. Anterior repression of a Drosophila stripe enhancer requires three position-specific mechanisms. Development. 2002;129:4931-40.

9. Vincent BJ, Estrada J, Depace AH. The appeasement of Doug a synthetic approach to enhancer biology. Integr Biol. 2016;8:475-84. 
10. Johnson LA, Zhao Y, Golden K, Barolo S. Reverse-engineering a transcriptional enhancer: a case study in Drosophila. Tissue Eng Part A 2008;14:1549-59.

11. Goto T, MacDonald P, Maniatis T. Early and late periodic patterns of even-skipped, expression are controlled by distinct regulatory elements that respond to different spatial cues. Cell. 1989;57:413-22.

12. Small S, Blair A, Levine M. Regulation of even-skipped, stripe 2 in the Drosophila embryo. EMBO J. 1992;11:4047-57.

13. Small S, Kraut R, Hoey T, Warrior R, Levine M. Transcriptional regulation of a pair-rule stripe in Drosophila. Genes Dev. 1991;5:827-39.

14. Stanojevic D, Small S, Levine M. Regulation of a segmentation stripe by overlapping activators and repressors in the Drosophila embryo. Science. 1991;254:1385-7.

15. Frasch M, Levine M. Complementary patterns of even-skipped, and fushi-tarazu, expression involve their differential regulation by a common set of segmentation genes in Drosophila. Genes Dev. 1987;1:981-95.

16. Surkova S, Myasnikova E, Janssens H, Kozlov KN, Samsonova A, Reinitz J, et al. Pipeline for acquisition of quantitative data on segmentation gene expression from confocal images. Fly. 2008;2:58-66.

17. Surkova S, Kosman D, Kozlov K, Manu, Myasnikova E, Samsonova A, et al. Characterization of the Drosophila, segment determination morphome. Dev Biol. 2008;313(2):844-62.

18. Luengo-Hendriks CL, Keranen SVE, Fowlkes CC, Simirenko L, Weber GH, Henriquez C, et al. 3D morphology and gene expression in the Drosophila blastoderm at cellular resolution I: data acquisition pipeline. Genome Biol. 2006;7:R123.

19. Fowlkes CC, Hendricks CLL, Keränen SVE, Rübel GHWO, Huang M, Chatoor S, et al. A quantitative spatiotemporal atlas of gene expression in the Drosophila blastoderm. Cell. 2008;133:364-74.

20. Janssens H, Hou S, Jaeger J, Kim AR, Myasnikova E, Sharp D, et al. Quantitative and predictive model of transcriptional control of the Drosophila melanogaster even skipped gene. Nat Genet. 2006;38:1159-65.

21. Segal E, Raveh-Sadka T, Schroeder M, Unnerstall U, Gaul U. Predicting expression patterns from regulatory sequence in Drosophila segmentation. Nature. 2008;451:535-40.

22. Samee MAH, Sinha S. Quantitative modeling of a gene's expression from its intergenic sequence. PLoS Comput Biol. 2014;10:1-21.

23. Kazemian M, Blatti C, Richards A, McCutchan M, Wakabayashi-Ito N, Hammonds AS, et al. Quantitative analysis of the Drosophila segmentation regulatory network using pattern generating potentials. PLoS Biol. 2010;8:e1000456.

24. He X, Samee MAH, Blatti C, Sinha S. Thermodynamics-based models of transcriptional regulation by enhancers: the roles of synergistic activation, cooperative binding and short-range repression. PLoS Comput Biol. 2010;6:e1000935.

25. Kim AR, Martinez C, Ramos AF, Ludwig MZ, Ogawa N, et al. Rearrangements of 2.5 kilobases of noncoding DNA from the Drosophila even-skipped locus define predictive rules of genomic cis-regulatory logic. PLoS Genet. 2013;9:e1003243.

26. Martinez C, Kim AR, Rest JS, Ludwig M, Kreitman M, White K, et al. Ancestral resurrection of the Drosophila S2E enhancer reveals accessible evolutionary paths through compensatory change. Mol Biol Evol. 2014;31:903-16

27. Sayal R, Dresch JM, Pushel I, Taylor BR, Arnosti D. Quantitative perturbation-based analysis of gene expression predicts enhancer activity in early Drosophila embryo. eLife. 2016;5:e08445.

28. Gray S, Szymanski P, Levine M. Short-range repression permits multiple enhancers to function autonomously within a complex promoter. Genes Dev. 1994;8:1829-38.

29. Gray S, Levine M. Short-range transcriptional repressors mediate both quenching and direct repression within complex loci in Drosophila. Genes Dev. 1996;10:700-10.

30. Fakhouri WD, Ay A, Sayal R, Dresch J, Dayringer E, Arnosti DN. Deciphering a transcriptional regulatory code: modeling short-range repression in the Drosophila embryo. Mol Syst Biol. 2010;6:34.

31. Ludwig MZ, Patel NH, Kreitman M. Functional analysis of eve stripe 2 enhancer evolution in Drosophila: rules governing conservation and change. Development. 1998;125:949-58.

32. Ludwig MZ, Palsson A, Alekseeva E, Bergman CM, Nathan J, Kreitman M. Functional Evolution of a cis-Regulatory Module. PLoS Biol. 2005;3(4):e93.
33. Hare EE, Peterson BK, Iyer VN, Meier R, Eisen MB. Sepsid even-skipped enhancers are functionally conserved in Drosopila despite lack of sequence conservation. PLoS Genet. 2008;4:e1000106.

34. Hare EE, Peterson BK, Eisen MB. A careful look at binding site reorganization in the even-skipped enhancers of Drosophila and Sepsids. PLoS Genet. 2008;4(11):e1000268.

35. Martinez CA, Barr KA, Kim AR, Reinitz J. A synthetic biology approach to the development of transcriptional regulatory models and custom enhancer design. Methods. 2013;62:91-98.

36. Groth AC, Fish M, Nusse R, Calos MP. Construction of transgenic Drosophila by using the site-specific integrase from phage phiC31. Genetics. 2004;166:1775-82.

37. Sackerson C, Fujioka M, Goto T. The even-skipped locus is contained in a 16-kb chromatin domain. Dev Biol. 1999;211:39-52.

38. Janssens $H$, Kosman D, Vanario-Alonso CE, Jaeger J, Samsonova M, Reinitz J. A high-throughput method for quantifying gene expression data from early Drosophila embryos. Dev Genes Evol. 2005;215:374-81.

39. Pisarev A, Poustelnikova E, Samsonova M, Reinitz J. FlyEx, the quantitative atlas on segmentation gene expression at cellular resolution. Nucleic Acids Res. 2008;37:D560-6.

40. Small S, Arnosti DN, Levine M. Spacing ensures autonomous expression of different stripe enhancers in the even-skipped promoter. Development. 1993;119:767-72.

41. Hertz GZ, Stormo GD. Identifying DNA and protein patterns with statistically significant alignments of multiple sequences. Bioinformatics. 1999;15:563-77.

42. Tamura K, Subramanian S, Kumar S. Temporal patterns of fruit fly (Drosophila) evolution revealed by mutation clocks. Mole Biol Evol. 2004;21:36-44.

43. Han K, Levine M, Manley JL. Synergistic activation and repression of transcription by Drosophila homeobox proteins. Cell. 1989;56:573-83.

44. Zhu LJ, Christensen RG, Kazemian M, Hull CJ, Enuameh MS, Basciotta MD, et al. FlyFactorSurvey: a database of Drosophila transcription factor binding specificities determined using the bacterial one-hybrid system. Nucleic Acids Res. 2011;39:D111-7.

45. Tomancak P, Beaton A, Weiszmann R, Kwan E, Shu S, Lewis SE, et al. Systematic determination of patterns of gene expression during Drosophila embryogenesis. Genome Biol. 2002;3(12):RESEARCH0088.

46. Chen H, Zhe X, Mei C, Yu D, Small S. A system of repressor gradients spatially organizes the boundaries of bicoid-dependent target genes. Cell. 2012;2:618-29.

47. Struffi P, Corado M, Kaplan L, Yu D, Rushlow C, Small S. Combinatorial activation and concentration-dependent repression of the Drosophila even skipped stripe 3+7 enhancer. Development. 2011;138:4291-9.

48. Tsurumi A, Xia F, Li J, Larson K, LaFrance R, Li WX. STAT is an essential activator of the zygotic genome in the early Drosophila embryo. PLoS Genet. 2011;72:e1002086.

49. Hanes SD, Riddihough G, Ish-Horowicz D, Brent R. Specific DNA recognition and intersite spacing are critical for action of the bicoid morphogen. Mol Cellular Biol. 1994;14:3364-75.

50. Satija R, Bradley RK. The TAGteam motif facilitates binding of 21 sequence-specific transcription factors in the Drosophila embryo. Genome Res. 2012;22:656-65.

51. Schulz KN, Bondra ER, Moshe A, Lieb JEVJD, Kaplan T, McKay DJ, et al. Zelda is differentially required for chromatin accessibility, transcription factor binding, and gene expression in the early Drosophila embryo. Genome Res. 2015;25:1715-26.

52. Fu D, Zhao C, Ma J. Enhancer sequences influence the role of the amino-terminal domain of Bicoid in transcription. Mol Cellular Biol. 2003;23:4439-48.

53. Burz DS, Rivera-Pomar R, Jaeckle H, Hanes SD. Cooperative DNA-binding by Bicoid provides a mechanism for threshold-dependent gene activation in the Drosophila embryo. EMBO J. 1998;17:5998-6009.

54. Maricque BB, Dougherty JD, Cohen BA. A genome-integrated massively parallel reporter assay reveals DNA sequence determinants of cisregulatory activity in neural cells. Nucleic Acids Res. 2017;45:e16.

55. Erceg J, Sauders TE, Girardot C, Devos DP, Hufnagel L, Furlong EEM. Subtle changes in motif positioning cause tissue-specific effects on robustness of an enhancer's activity. PLoS Genet. 2014;10:e1004060.

56. Kulkarni MM, Arnosti DN. cis-Regulatory logic of short-range transcriptional repression in Drosophila melanogaster. Mol Cellular Biol. 2005;25:3411-20. 
57. Smith RP, Taher L, Patwardhan RP, Kim MJ, Inoue F, Shendure J, et al. Massively parallel decoding of mammalian regulatory sequences supports a flexible organizational model. Nat Genet. 2013;45:1021-8.

58. Innocentini GCF, Hornos JEM. Modeling stochastic gene expression under repression. J Math Biol. 2007;55:413-31.

59. Prata GN, Hornos JE, Ramos AF. Stochastic model for gene transcription on Drosophila melanogaster embryos. Phys Rev E. 2016;93:022403.

60. He BZ, Holloway AK, MaerkI SJ, Kreitman M. Does positive selection drive transcription factor binding site turnover? A test with Drosophila cis-regulatory modules. PLoS Genet. 2011;7:e1002053.

61. Gibson D. One-step enzymatic assembly of DNA molecules up to several hundred kilobases in size. Protocol Exchange. 2009. doi:10.1038/nprot.2009.77.

62. Ramos AF, Innocentini GCP, Forger FM, Hornos JEM. Symmetry in biology: from genetic code to stochastic gene regulation. IET Syst Biol. 2010;4:311-29.

\section{Submit your next manuscript to BioMed Central and we will help you at every step:}

- We accept pre-submission inquiries

- Our selector tool helps you to find the most relevant journal

- We provide round the clock customer support

- Convenient online submission

- Thorough peer review

- Inclusion in PubMed and all major indexing services

- Maximum visibility for your research

Submit your manuscript at www.biomedcentral.com/submit 